\title{
Incorporation of Radio Frequency Identification Tag in Dentures to Facilitate Recognition and Forensic Human Identification
}

\author{
E. Nuzzolese ${ }^{1, *}$, V. Marcario ${ }^{2}$ and G. Di Vella ${ }^{3}$ \\ ${ }^{1}$ Forensic Odontologist, Bari, Italy \\ ${ }^{2}$ Dental Technician, Bitritto, (Bari), Italy \\ ${ }^{3}$ Associate Professor in Forensic Pathology, Sezione di Medicina Legale, Università degli Studi “A. Moro”, Bari, Italy
}

\begin{abstract}
Forensic identification using odontology is based on the comparison of ante-mortem and post mortem dental records. The insertion of a radio frequency identification (RFId) tag into dentures could be used as an aid to identify decomposed bodies, by storing personal identification data in a small transponder that can be radio-transmitted to a reader connected to a computer. A small passive, $12 \times 2,1 \mathrm{~mm}$, read-only RFId-tag was incorporated into the manufacture of three trial complete upper dentures and tested for a signal. The aim of this article is to demonstrate the feasibility of manufacturing such a dental prosthesis, the technical protocols for its implantation in the denture resin and its working principles. Future research and tests are required in order to verify human compatibility of the tagged denture and also to evaluate any potential deterioration in strength when subjected to high temperatures, or for damage resulting from everyday wear and tear. It should also be able to withstand the extreme conditions resulting from major accidents or mass disasters and procedures used to perform a forensic identification.
\end{abstract}

Keywords: Forensic dentistry, denture labelling, radio-frequency identification, human identification, forensic science.

\section{INTRODUCTION}

RFId technology was first introduced in 1940, during World War II, and used to identify aeroplanes belonging to the Royal Air Force [1]. During the 1980's and 1990's, with the advances in information technology and the possibility of producing low cost tags the interest in RFId was renewed. It forms part of a technology known as "automatic identification and data capture" and is used to identify, locate and track people, animals and property [2,3].

The acronym RFID stands for radio-frequency identification, which is a wireless electronic communication technology [4]. A serial number that identifies a person, animal, or object is stored in a microchip with an attached antenna. The chip and antenna together are called an RFID-tag or transponder (Fig. 1). The antenna enables the chip to transmit the serial number, or other information to a reader. The reader converts the radio waves reflected back from the RFID-tag into digital information that are then passed to a computer with applications to interpret it. There are 'passive' tags, so named because the power for reading the information on the chip is sent from the reader. 'Active' tags on the other hand, carry their own power supply enabling them to communicate between tag and reader, thus increasing the potential for detection of the signal. Another feature of RFId-tags is a $\mathrm{read} / \mathrm{write}$ function. There are 'read only' and 'read/writable' chips, to which new data can be transmitted. Tags come in various forms and dimensions, and can be adapted to specific applications. Every transponder has an unchangeable unique

*Address correspondence to this author at Ambulatorio Nuzzolese, Viale JF Kennedy 77, 70124 Bari, Italy; Tel: +39 080 5042555; Fax: +39 080 22031198; E-mail: emilionu@tin.it identification number, allowing individual tags to be identified within a group. Many of the physical properties of RFID-systems and their influence on biological materials depend on the frequency used to transmit the data [5] (Table 1).

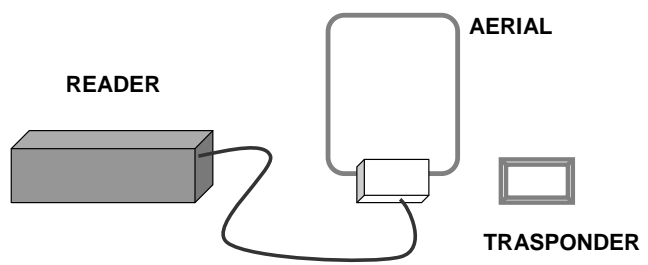

Fig. (1). RFID transponder: include a chip and an antenna.

In the healthcare sector the use of the RFId-tags has been tested for suitability in various fields, such as transfusion medicine [6] replacing the bar code with a tag. In Italy at the Ophthalmologic department of Niguarda Hospital (Milan) patients wear a microchip bracelet which stores all the medical data relevant to the surgery they will be undergoing [7]. In the last 2-3 years, the use of RFID has been applied in areas previously not considered suitable. Trials to evaluate potential applications for RFID in health care are ongoing in areas such as: access control and logging employee working times; regulation of access to elevators; protection of expensive equipment; location of patients, staff and equipment in hospitals and nursing homes; organisation of logistic processes for containers, apparel, beds; safe identification of patients and materials; protection against counterfeit drugs.

There are other possible applications of this technology are proposed in the forensic field [8]. Recent studies have investigated the use of an RFId for body tagging and management of resources at a hospital during a mass casualty 
Table 1. Frequencies Used for RFID, Read Distances and Some Typical Applications

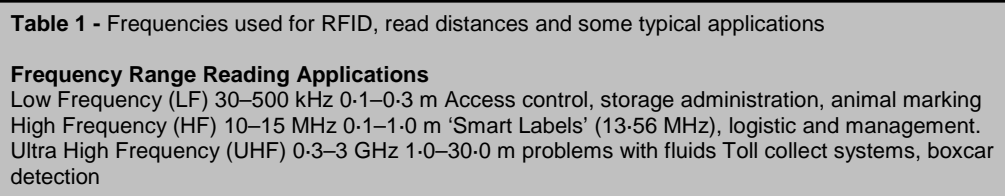

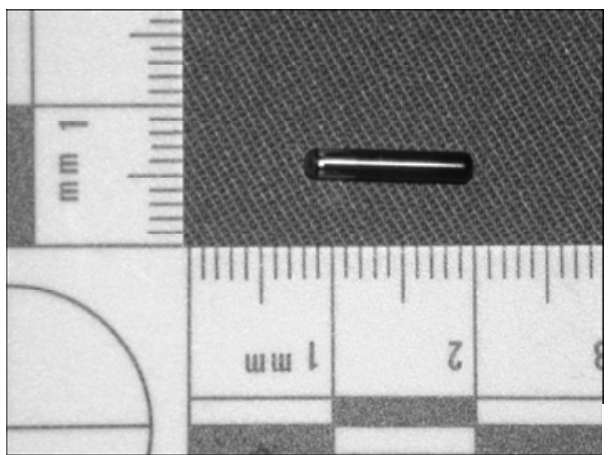

Fig. (2). Passive, $10 \mathrm{~mm}$ length, torpedo shaped, read-only low frequency tag.

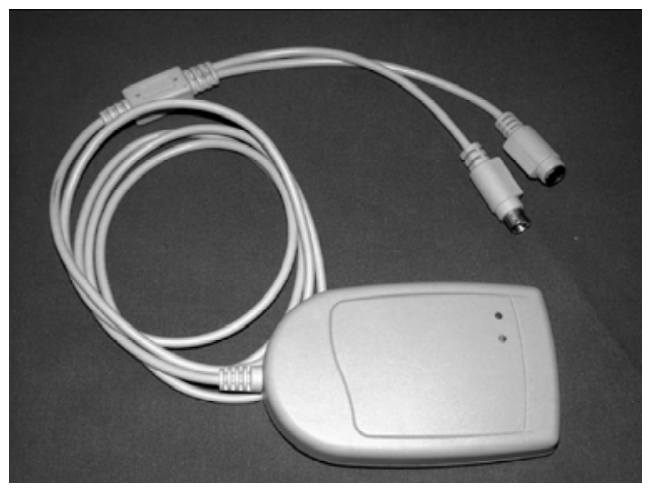

Fig. (3). RFId tag Reader to be connected to a computer in the keyboard jack.

situation, such as during the tsunami disaster in 2004 by the Austrian DVI team [9]. The RFId technology can be also employed as an aid for forensic dental identification based on the comparison of ante-mortem and post-mortem dental records, by placing a small transponder in teeth $[10,11]$ and dental prostheses [12,13]. Determining the various individual characteristics of the human dentition has proved an effective aid in the task of identification. Edentulous people on the other hand, have lost all or most of the key features that have proven valuable in such cases, hence the identification process is made much more difficult unless the victims wear marked dentures [14]. Over the years various denturemarking systems have been trialled $[15,16]$. These can be divided into surface marking and inclusion methods. Inclusion methods involve the incorporation of metallic or nonmetallic labels, barcodes [17] or microchips [18, 19]. The RFId small transponder has not been popular owing to its high cost and relative unavailability. An attempt was made therefore to develop a denture marking system which improves on previous systems in terms of its simplicity, cost

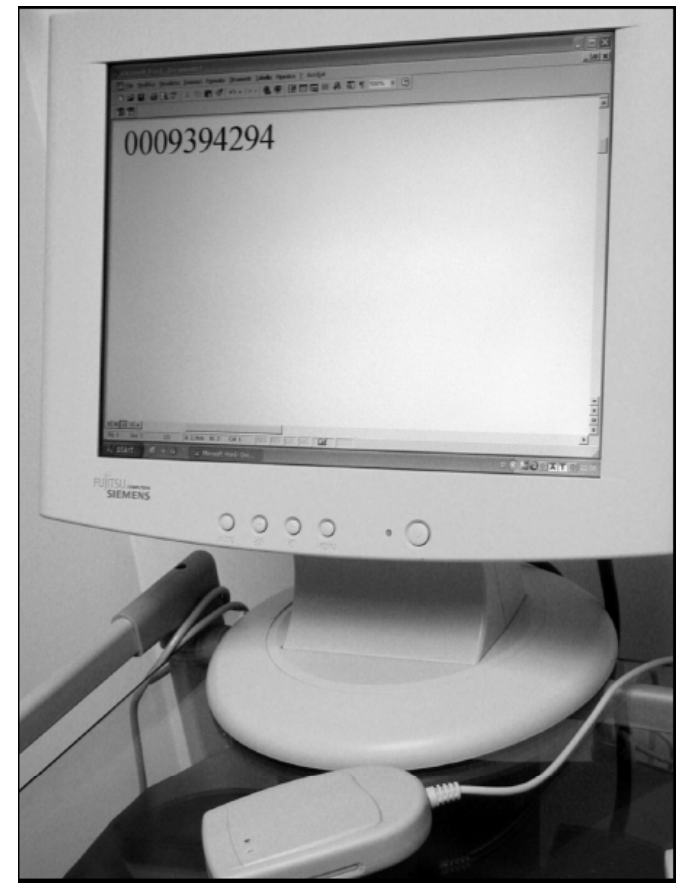

Fig. (4). Signal transmitted displayed on the pc monitor under a word processor.

and effectiveness, with particular emphasis on the amount of storable data.

The information obtained from this study should be considered as a preliminary test of RFId-tags for forensic deployment, and give some indications as to optimum design, choice and implantation of the ideal tag in a complete upper denture.

\section{EQUIPMENTS AND MATERIALS}

The tags used in this experiment were provided by an Italian RFId manufacturer (MRFID Network). Passive, 12 mm length $X 2,1 \mathrm{~mm}$ width, torpedo shaped, read-only low frequency tags were chosen (Fig. 2). Only low frequencies, $125-134 \mathrm{kHz}$, are employed for veterinary use and are also acceptable for human use. The small RFId-tag was incorporated into three sample complete upper dentures. No special training or a dental technician is required and the device incorporation can be made in the dental office.

The transponder contains no batteries and is hermetically sealed in a protective tube. The reader energizes the transponder by means of an electromagnetic field emitted via the reader's antenna. The reader is connected to a computer in the keyboard jack and the signal is displayed on the pc monitor using a word processor (Figs. 3-4). 




Fig. (5). $12 \mathrm{~mm} \times 3 \mathrm{~mm}$ in depth depression is made in the denture's external surface with a carbide bur.

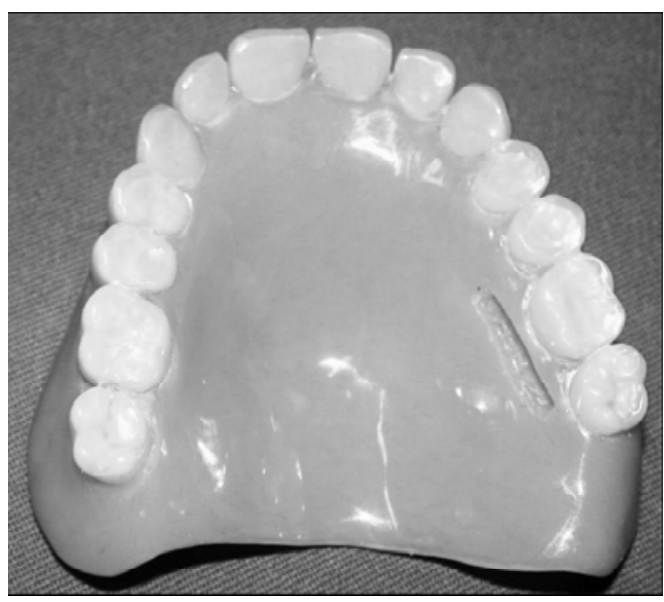

Fig. (6). Depression prepared in the denture ready for the tag incorporation.

\section{PROCEDURES AND RESULTS}

A $12 \mathrm{~mm}$ X $3 \mathrm{~mm}$ in depth depression was made in the denture's external surface with a carbide bur (Figs. 5-7). This dimension is required to incorporate the tag without structurally weakening the denture. The tags were positioned so that their long axes were placed parallel to occlusal plane, with the antenna orientated mesially. The tag was covered with pink auto polymerizing acrylic resin (Kulzer HerausKulzer, Germany) eliminating any trapped air bubbles. The denture was then processed in a pressurized container with warm water $\left(40^{\circ}, 25 \mathrm{psi}\right)$, and was finally finished and polished (Fig. 8). The three RFId-tag implanted dentures were tested to verify the efficacy and range of data transmission. The reader sends a coded signal that is returned by the transponder, and then converts it into readable data. The tag was visible even when embedded in pink acrylic resin. The read out was positive. The optimum read-out position in the in vitro test was achieved with the reader perpendicular to the long axis of the microchip, with a maximum scanning range c. $1 \mathrm{~cm}$.

\section{DISCUSSION}

The object of this article is to demonstrate the feasibility in the manufacture of a dental prosthesis with an incorpo-

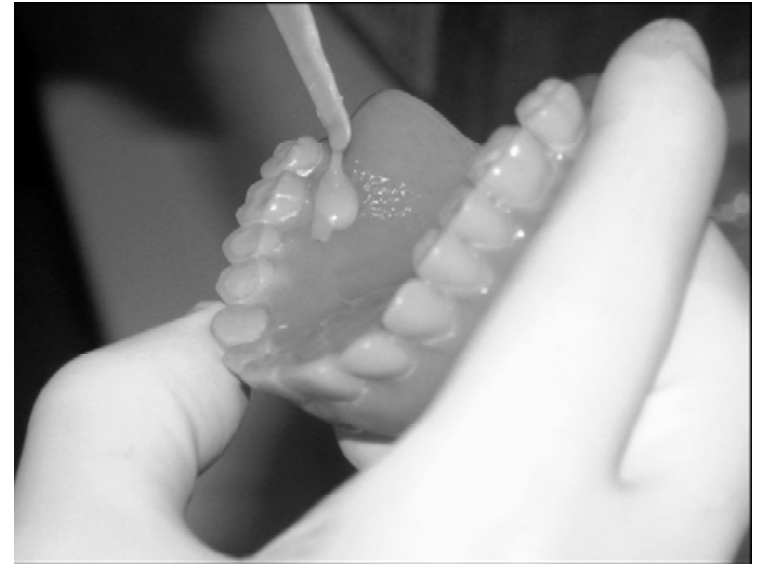

Fig. (7). The tag is covered with pink auto polymerizing acrylic resin.

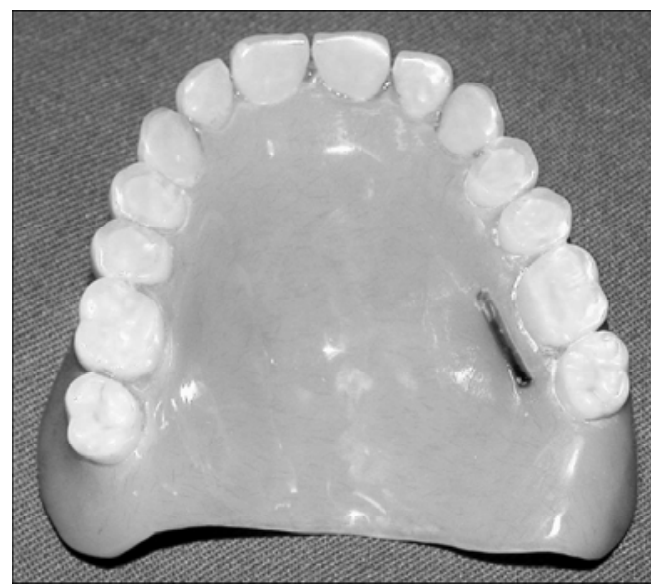

Fig. (8). Denture, with incorporated, RFId finished and polished.

rated radio frequency identification tag, the technical procedures for its incorporation in the denture resin and its working principles. There are several advantages in using this labeling system. The dentist without requiring special training or a dental technician can easily set the tag in the denture, since every dental surgery is equipped to carry out prosthetic adjustments and has auto polymerizing acrylic resin. Because of the tag's size there is no real weakening of the denture as would be expected with metallic markers. Should the patient need laboratory reline or rebase there is no need to remove the device from the denture before reline/rebase and finishing procedure.

Finally, the cost for an RFId tag is nowadays around US\$ 20 , making the procedure a viable option. Estimates suggest that the cost of "smart labels" will reduce further making the procedure extremely cost-effective. It is also worth mentioning that, a European sanitary regulation, European directive 9342C implemented in 1993, requires that prosthesis provided by a laboratory to a dentist be accompanied by a record, either paper or digital, which includes the history of manufacture of the item.

As with any new technology, data protection and privacy issues must necessarily be considered. The results obtained from our in vitro test, and in other similar experiments [20] indicate that it would be extremely unlikely that patient pri- 
vacy could be compromised, owing to the very close proximity of the reader to the microchip before a signal can be obtained.

\section{CONCLUSION}

A general dentist without any special training or assistance from a laboratory technician can easily perform the incorporation of a microchip transponder in a denture and the procedure is extremely economical. Its inclusion offers many benefits in dental prosthetic labeling; allowing not only the storage of patient's medical records on a searchable database, but also information on the materials used and so providing traceability. Ultimately the chip could play an invaluable forensic role in assisting with human identification. It is however, reasonable to conclude that further investigation is needed to evaluate RFId-tags technical performance under a range of post-mortem trauma such as high temperatures and impact other environmental conditions.

\section{ACKNOWLEDGMENT}

The authors would like to thank MRFID Italia for the sample RFId tags donated for the purpose of this article.

\section{REFERENCES}

[1] Landt J. Shrouds of Time, the History of RFID. AIM Publication 2001, available at: http://www.aimglobal.org

[2] Finkelzeller K. The RFID handbook, $2^{\text {nd }}$ ed, New York: John Wiley \& Sons 2003.

[3] Want R. An Introduction to RFID technology. IEEE Pervasive Comput 2006; 5(1): 25-33.

[4] Kabachinski J. An introduction to RFID. Biomed Instrum Technol 2005; 39(2): 131-4.

[5] Spekman RE, Sweeney II PJ. RFID: from concept to implementation. Int J Phys Dist Logist Manage 2006; 36(10): 736-54.

[6] Knels R. Radio frequency identification (RFID): an experience in transfusion medicine. ISBT Sci Series 2006; 1: 238-41.
[7] Rusconi G. Tag at the patients wrists in niguarda hospital in milan (article in Italian) 2006; Week.it: Available at www.weekit.it

[8] Fry EA, Lenert LA. MASCAL: RFID tracking of patients, staff and equipment to enhance hospital response to mass casualty events. AMIA Annu Symp Proc 2005; pp. 261-5.

[9] Meyer HJ, Chansue N, Monticelli F. Implantation of radiofrequency identification device (RFID) microchip in disaster victim identification (DVI). Forensic Sci Int 2006; 157(2-3): 168-71.

[10] Thevissen PW, Poelman G, De Cooman M, Puers R, Willems G. Implantation of an RFID-tag into human molars to reduce hard forensic identification labor. Part 1: Working principle. Forensic Sci Int 2006; 159(Suppl 1): S33-9.

[11] Thevissen PW, Poelman G, De Cooman M, Puers R, Willems G. Implantation of an RFID-tag into human molars to reduce hard forensic identification labor. Part 2: physical properties. Forensic Sci Int 2006; 159(Suppl 1): S40-6.

[12] Millet C, Jeannin C. Incorporation of microchips to facilitate denture identification by radio frequency tagging. J Prosthet Dent 2004; 92(6): 588-90.

[13] Ilic C. Using tags to make teeth. RFID J 2004. Available at www.rfidjournal.com/article/articleview/1206/1/1/

[14] Bengtsson A, Olsson T, Rene N, Carlsson GE, Dahlbom U, Borrman $\mathrm{H}$. Frequency of edentulism and identification marking of removable dentures in long-term care units. J Oral Rehabil 1996; 23(8): 520-3

[15] Rajan M, Julian R. A new methods of making dentures using microchips. J Forensic Odontostomatol 2002; 20(1): 1-5.

[16] Richmond R, Pretty IA. Contemporary methods of labelling dental prostheses - a review of literature. J Forensic Sci 2006; 51(5): $1120-6$.

[17] Milward PJ, Shepherd P, Brickley MR. Automatic identification of dental appliances. Br Dent J 1997; 182: 171-4.

[18] Bernitz H, Blignaut J. An inclusion technique for marking denture. J Foren Odontostomatol 1998; 16: 14-6.

[19] Rotzscher K, Bedrich MR, Jurisch R, Peitsch P. Electronic denture marking. J Forensic Odontostomatol 1999; 17: 27-9.

[20] Richmond R, Pretty IA. The use of radio-frequency identification tags for labeing dentures - scanning properties. J Forensic Sci 2009 54(3): 664-8

Received: September 12, 2009

(C) Nuzzolese et al.; Licensee Bentham Open .

This is an open access article licensed under the terms of the Creative Commons Attribution Non-Commercial License (http://creativecommons.org/licenses/by-nc/3.0/) which permits unrestricted, non-commercial use, distribution and reproduction in any medium, provided the work is properly cited. 